Results In our study we have observed a greater incidence of preterm birth (33\%) and caesarian section (68\%), compared to reference healthy population of the same hospital Department. We also observed, in association with maternal therapy taken during pregnancy, a significant difference of the incidence of preterm birth $(p=0,0005)$ and a weakly significant difference of the incidence of low neonatal weight $(\mathrm{p}=0,0596)$.

We haven't notice any significant difference, in association with antibody positivity, of the incidence of low birth weight and fetal growht restriction and not even in the incidence of neoantal complications.

Regarding the long-term paediatric follow-up, we have notice a greater incidence of psycomotor development alterations in association with ENA antibody positivity $(28 \%$ vs $0 \%)$, with a statistically significant difference $(p=0.0155)$.

Conclusions Data from the retrospective analysis, in agreement with literature, confirm a greater incidence of caesarian section (most of cases programmed), preterm birth and low birth weight in this category of newborns.

For the first time it was considered the association between neonatal and paediatric outcomes and the maternal tharapy during pregnancy: we have notice a significant greater incidence of preterm birth in newborns from mother who have taken therapy during pregnancy.

Regarding the long-term paediatric follow-up, until school age, in agreement with the few data in the literature, it was observed a greater incidence of psychomotor development alterations in children born from mother with antibody positivity (aPL ed ENA).

Our data notice a poor adherence to practical recommendations of the clinical management and the follow-up of these children present in the literature.

We have set up a national survey in order to verify current knowledge and to sensitise Paediatricians and Neonatologists regarding this category of children, that need a specific clinical management and follow-up.

Since limits of a retrospecive study and the relative small population size, we started a case-control prospective study with a wider population size, currently in the recruitment phase, in order to validate data observed in the retrospective analysis and to verify the current adhesion to raccomandations of the follow-up.

We hope that a greater knowledge of the topic, together with the establishment of common clinical practices, can bring to an improvement of obstetric, neonatal and long-term paediatric outcomes in children born from mother with systemic autoimmune disease.

\section{PS4:84 OBSTETRICAL COMPLICATIONS AND PREGNANCIES IN WOMEN WITH SYSTEMIC LUPUS ERYTHEMATOSUS}

T Ben Salem, M Tougorti, I Naceur, M Lamloum, I Ben Ghorbel, MH Houman. Department of Internal Medicine, La Rabta University Hospital, Tunis, Tunisia

\subsection{6/lupus-2018-abstract.129}

Background Systemic lupus erythematosus (SLE) is a chronic auto-immune disease, which mainly occurs in women during childbearing age. It is associated with several fetal and maternal complications.

Objective To describe obstetrical complications and evaluate pregnancy and fecundity rates in women with SLE.
Methods It is a retrospective study including 227 women with SLE. Only 143 women, who are sexually active, were studied. Results The mean age at disease onset was $36.61 \pm 13.41$ years. The mean age at SLE diagnosis was $38.47 \pm 13.43$ years. The most frequent manifestations of the disease were lupus nephritis and neurological involvements, seen in respectively $39.7 \%$ and $14.7 \%$ of patients. Antinuclear antibodies were positive in $96.5 \%$ of cases and anti-dsDNA were positive in $96.3 \%$ of cases. Anti-SSA, anti-SSB, anti-cardiolipin and anti-B2GPI antibodies were found in respectively $68,37.3,46 \%$ and $27.3 \%$ of patients. Corticosteroids were given in $90.4 \%$ of patients and 58\% of them had immunosuppressive therapy: Cyclophosphamide $(n=42)$, Azathioprine $(n=30)$, Methotrexate $(n=9)$ and Mofetil Mycophenolate $(n=2)$.

Obstetrical complications were: spontaneous abortion before the $10 \mathrm{TH}$ week of gestation (22.8\%), unexplained fetus death after the $10 \mathrm{TH}$ week of gestation (12.2\%) and intrauterine death (10\%).

Among the 143 women, 130 had at least one pregnancy (average $=3.59 \pm 2.62$ pregnancies). Most pregnancies resulted in live births; 121 women had at least one child with an average of $2.55 \pm 2$ children/woman. Pregnancies occurred after SLE diagnosis in 40 women (among 69 who wants to have children) with a mean of $1.75 \pm 1.05$ pregnancies. Twenty nine women had at least one child with a mean of $1.15 \pm 0.9$ children. In addition to their usual treatment, other drugs were added during pregnancies: aspirine $(n=7)$, low molecular weight heparin $(n=7)$ and betamethasone in one case. One patient was treated with aspirine and low molecular weight heparin. Obstetrical events caused lupus flare in 11 cases (pregnancy in 7 cases, delivery in 2 cases, intrauterine death and spontaneous abortion each in one case).

Conclusion Comparing to general population in Tunisia (2 children/woman), fecundity is reduced in women after SLE diagnosis. Complications are related to disease activity, antiphophspholipid antibodies and hypertension.

\section{PS4:85 FERTILITY AND PREGNANCY IN WOMEN WITH SYSTEMIC LUPUS ERYTHEMATOSUS: A COMPARISON WITH OTHER RHEUMATIC DISEASES}

${ }^{1}$ IC Chalmeta-Verdejo, ${ }^{1} \mathrm{~K}$ Arevalo, ${ }^{1} \mathrm{~J}$ Ivorra, ${ }^{2} \mathrm{C}$ Diaz-García, ${ }^{1} \mathrm{R}$ Negueroles, ${ }^{1} \mathrm{E}$ Grau, ${ }^{1} \mathrm{~L}$ Gonzalez-Puig, ${ }^{1}$ I Martinez-Cordellat, ${ }^{1} \mathrm{C}$ Feced, ${ }^{1} \mathrm{~F}$ Ortiz, ${ }^{1} \mathrm{C}$ Alcañiz, ${ }^{1} \mathrm{E}$ Vicens, ${ }^{1} \mathrm{~J}$ Oller, ${ }^{1} E$ Labrador, ${ }^{1} \mathrm{~J}$ Fragio, ${ }^{1} \mathrm{R}$ González, ${ }^{1} \mathrm{M}$ de la Rubia, ${ }^{1} \mathrm{C}$ Nájera, ${ }^{1} \mathrm{I}$ Canovas, ${ }^{1} \mathrm{~J}$ A RománIvorra. ${ }^{1}$ Rheumatology Department. Hospital Universitario la fe, Valencia, Spain; ${ }^{2}$ Unidad de Preservacion de Fertilidad, Hospital Universitario la fe, Valencia, Spain

\subsection{6/lupus-2018-abstract.130}

Purpouse Rheumatological disorders, usually affect women during reproductive ages and have an impact on their reproductive wishes.

We aim to assess the differences between SLE and other diseases for fertility and pregnancy evolution in a group of patients followed in a specific consult for planning and management of pregnancy.

Methods Retrospective study in a cohort of patients with rheumatological disorders who want to get pregnant. Fertility exam to the couple was done. Data about sterility (never conception) and infertility (miscarriages), assisted reproduction, preterm delivery and also adverse pregnancy outcomes (disease flares, preeclampsia) were compared in 3 groups: SLE, connective tissue disease non-SLE (Sjögren's Syndrome, MCTD) and 